\title{
A NEW AND CONSTRUCTIVE PROOF OF THE BORSUK-ULAM THEOREM
}

\author{
MARK D. MEYERSON AND ALDEN H. WRIGHT
}

\begin{abstract}
The Borsuk-Ulam Theorem [1] states that if $f$ is a continuous function from the $n$-sphere to $n$-space $\left(f: S^{n} \rightarrow \mathbf{R}^{n}\right)$ then the equation $f(x)=f(-x)$ has a solution. It is usually proved by contradiction using rather advanced techniques. We give a new proof which uses only elementary techniques and which finds a solution to the equation. If $f$ is piecewise linear our proof is constructive in every sense; it is even easily implemented on a computer.
\end{abstract}

The Borsuk-Ulam Theorem has applications to fixed-point theory and corollaries include the Ham Sandwich Theorem and Invariance of Domain. The method used here is similar to Eaves [2] and Eaves and Scarf [3].

We use the following notational conventions. Let $S^{n}=\left\{x=\left(x_{0}, \ldots, x_{n}\right)\right.$ $\in \mathbf{R}^{n+1} \mid$ some $\left.x_{i}= \pm 1\right\}$, the boundary of a cube. Note that the antipodal map $\alpha: S^{n} \rightarrow S^{n}$, defined by $\alpha(x)=-x$, is a PL homeomorphism. We use $s, t \in \mathbf{R} ; p, p^{\prime}, z \in \mathbf{R}^{n} ; x, y \in S^{n} \subset \mathbf{R}^{n+1} ; v \in S^{n} \times I \subset \mathbf{R}^{n+2} ;$ and by tuples such as $(p, s)$ or $(z, s, t)$ we mean the obvious points of $\mathbf{R}^{n+1}$ or $\mathbf{R}^{n+2}$. A singleton set, such as $\{t\}$, will be represented without brackets, $t$. The origin in $\mathbf{R}, \mathbf{R}^{n}$, and $\mathbf{R}^{n+1}$ will be represented by 0 . We will let $G_{t}(x)=$ $G(x, t)$.

The Piecewise Linear Borsuk-Ulam Theorem. Let $f: S^{n} \rightarrow \mathbf{R}^{n}$ be $a$ PL map. Then there exists an $x \in S^{n}$ such that $f(x)=f(-x)$.

Proof. Since $f$ is PL, it is linear on each simplex of a triangulation $T$ of $S^{n}$. Let $T \cap \alpha T$ denote the subdivision of $T$ into convex cells obtained by intersecting each simplex of $T$ with the image of a simplex of $T$ under $\alpha$. Then $f$ is linear on each cell of $T \cap \alpha T$ and $T \cap \alpha T$ is invariant under $\alpha$. We can subdivide $S^{n} \times I$ into convex cells by crossing each cell of $T \cap \alpha T$ with $I$.

We next subdivide these convex cells without adding new vertices to get a triangulation $T^{*}$ which is still invariant under the homeomorphism

$$
H=\alpha \times \text { id: } S^{n} \times I \rightarrow S^{n} \times I
$$

$(H(x, t)=(-x, t))$. To do this, order the pairs of vertices $\{v, H(v)\}$. Note

Received by the editors November 22, 1977 and, in revised form, March 16, 1978.

AMS (MOS) subject classifications (1970). Primary 55C20, 57C99, 57A15, 57A10; Secondary $90 C 99$.

Key words and phrases. Borsuk-Ulam Theorem, Ham Sandwich Theorem, Invariance of Domain, fixed point, antipodal map, piecewise linear, simplicial methods, complementary pivot theory. 
that $v$ and $H(v)$ cannot lie in a single convex cell of the subdivision. Suppose we have subdivided the $(r-1)$-skeleton. Let $C$ be an $r$-cell and let $v$ be a vertex of $C$ from the first vertex pair meeting $C$. Use $v$ to cone on the faces of $C$ not containing $v$. (See [5, Problem 2.9] for the details of this argument.)

Choose $p \in \mathbf{R}^{n}$ so that $(p, 1,1)$ lies in the interior of an $n$-simplex of $T^{*}$. Let $G_{0}(x)=f(x)-f(-x)$, for all $x \in S^{n}$, and let $G_{1}(z, s)=z-s p$ for all $(z, s) \in S^{n}$. Extend piecewise linearly to $G: S^{n} \times I \rightarrow \mathbf{R}^{n}$ using $T^{*}$. Note that $G(x, t)=-G(-x, t)$ and that $G_{1}^{-1}(0)=\{(p, 1),(-p,-1)\}$.

If $G\left(\sigma-S^{n} \times 0\right)$ contains 0 for any $(n-1)$-simplex $\sigma$ of $T^{*}$, we make the following adjustments in $G$ (otherwise take $p^{\prime}=p$ ). Make no change in $G \mid S^{n} \times 0$. Adjust the values of $G$ simultaneously on each pair $(z, s, 1)$ and $(-z,-s, 1)$ of vertices of $T^{*}$, redefining $G$ by extending piecewise linearly using $T^{*}$, so that:

(a) $G(z, s, 1)=-G(-z,-s, 1)$, for all $(z, s, 1) \in S^{n} \times 1$,

(b) For some $p^{\prime} \in \mathbf{R}^{n}, G_{1}^{-1}(0)=\left\{\left(p^{\prime}, 1\right),\left(-p^{\prime},-1\right)\right\}$, and

(c) No $G\left(\sigma-S^{n} \times 0\right)$ contains 0 , for any $\sigma$ in $T^{*}$ of dimension at most $n-1$.

(b) is achieved by making the change in $G$ small. For (c), suppose that we are adjusting at $v$ and $\sigma^{*} v$ contradicts (c) while $\sigma$ satisfies (c). Then any adjustment of $G(v)$ out of the plane determined by $G\left(\sigma^{*} v\right)$ will make $\sigma^{*} v$ satisfy (c).

Now let $A$ be the component of $G^{-1}(0)-\left(S^{n} \times 0\right)$ containing $\left(p^{\prime}, 1,1\right) . A$ is a polygonal arc which has its other endpoint either in $S^{n} \times 1$ or $S^{n} \times 0$ (in the latter case $A$ does not contain this endpoint). Then since $G(x, t)=$ $-G(-x, t), H(A)$ will be the component of $G^{-1}(0)-\left(S^{n} \times 0\right)$ containing $\left(-p^{\prime},-1,1\right)$. Either $A=H(A)$ or $A \cap H(A)=\phi$. The latter case holds since otherwise $A$ is a closed arc and $H$ would have to have a fixed point (by a PL version of the Intermediate Value Theorem). Hence $\operatorname{cl}(A)$ must be an arc connecting $S^{n} \times 1$ to $S^{n} \times 0$. So $\operatorname{cl}(A) \cap\left(S^{n} \times 0\right)$ is a solution.

Thus the algorithm for finding a solution consists of following a polygonal arc in $G^{-1}(0)$ from $S^{n} \times 1$ to $S^{n} \times 0$. This algorithm can be implemented numerically using techniques similar to those used to implement the simplex method of linear programming. See [2] for details. In practice, the adjustment of $G$ could be done in the process of following the arc. When the arc is found to intersect a simplex of dimension less than $n$, then $G$ could be adjusted to remove the intersection.

Corollary (The Borsuk-Ulam Theorem). Let $f: S^{n} \rightarrow \mathbf{R}^{n}$ be any continuous map. Then there exists an $x \in S^{n}$ so that $f(x)=f(-x)$.

Proof. Define $f^{k}: S^{n} \rightarrow \mathbf{R}^{n}$ by taking a triangulation of $S^{n}$ of mesh less than $1 / k$, setting $f^{k}(x)=f(x)$ at the vertices of the triangulation and extending linearly. Then $f^{k} \rightarrow f$ uniformly, and there exists $x_{k} \in S^{n}$ so that $f^{k}\left(x_{k}\right)=f^{k}\left(-x_{k}\right)$. It follows that a subsequence of $\left\{x_{k}\right\}$ converges to some $x$ and $f(x)=f(-x)$. 
One cannot hope to generalize this result much by changing the antipodal map. For Pannwitz [4] gives an example in which $\gamma: S^{n} \rightarrow S^{n}$ is a homeomorphism isotopic to the antipodal map which takes antipodal points to antipodal points and there is no solution to $f(x)=f(\gamma(x))$. In fact, by changing $-\beta|\beta|$ to $-\beta|\beta|^{\varepsilon}, \varepsilon>0$ at the bottom of p. 184 of [4], $\gamma$ still has the above properties and can also be made arbitrary close to the antipodal map.

ADDED IN PROOF. J. C. Alexander and J. A. Yorke have independently found a constructive proof of the Borsuk-Ulam Theorem. Their result is contained in the paper The homotopy continuation method: numerically implementable topological procedures, Trans. Amer. Math. Soc. (to appear).

\section{REFERENCES}

1. Karol Borsuk, Drei Sätze über die n-dimensional euklidische Sphäre, Fund. Math. 20 (1933), 177-190.

2. B. Curtis Eaves, A short course in solving equations with PL homotopies, Nonlinear programming (Proc. Sympos., New York, 1975), SIAM-AMS Proc., Vol. IX, Amer. Math. Soc., Providence, R.I., 1976, pp. 73-143.

3. B. Curtis Eaves and H. Scarf, The solution of systems of piecewise linear equations, Mathematics of Operations Research 1 (1976), 1-27.

4. E. Pannwitz, Eine freie Abbildung der n-dimensional Sphäre in die Ebene, Math. Nachr. 7 (1952), 183-185.

5. Colin P. Rourke and Brian J. Sanderson, Introduction to piecewise-linear topology, SpringerVerlag, Berlin and New York, 1972.

Department of MATHEmatics, University of Illinois, Urbana, Illinois 61801

Department of Mathematics, Western Michigan University, Kalamazoo, Michigan 49008

Current address (M. D. Meyerson): Department of Mathematics, U.S. Naval Academy, Annapolis, Maryland 21402

Current address (A. H. Wright): 700 Warren Road, Apartment 16-2A, Ithaca, New York 14850 